\title{
Use of therapeutic apheresis as a treatment method
}

Galhena $\mathrm{J}^{1}$, Jayasinghe $\mathrm{YARP}^{2}$, Abeygunarathne $\mathrm{G}^{3}$

Transfusion medicine has progressed beyond mere blood transfusion services. There are therapeutic modalities involving transfusion medicine less known to clinicians at large. What is broadly known as "plasmapheresis" is used to treat many clinical conditions including Guillain-barre syndrome, myasthenia gravis, chronic inflammatory demyelinating polyneuropathy, hyperviscosity in monoclonal gammopathies and thrombotic thrombocytopenic purpura. Proper term for this treatment modality is therapeutic apheresis.

"Apharesis" literallymeans "takingaway". Therapeutic apheresis includes therapeutic plasma exchange (TPE) and cytapheresis. Therapeutic cytapheresis removes cellular components from blood, returning plasma back into the circulation. Cytapheresis includes erythrocytapheresis, commonly known as red cell exchange which reduces abnormal red cell and leucopheresis reduces dangerously high white cell count.

Whereas, TPE effectively removes large molecular weight substancees from the plasma, such as monoclonal paraproteins and autoantibodies. In TPE, the plasma is removed while a replacement fluid is mixed with the cellular components and returned to the patient.

TPE can be performed using either centrifugation or filtration-based devices. Centrifugation separates blood components based on density of the individual elements. Filtration-based devices use membranes that contain pores that allow passage of proteins but not the cellular components. Once the plasma has been separated from the cells, it can either be discarded with the volume replaced or it can be further processed by the use of a second filter, the plasma fractionator (PF) to pass through the membrane to be returned to the patient. In membrane plasma filtration selectively remove undesired macromolecules, which then allow return of the processed plasma to the patient instead of donor plasma or albumin.

\section{Consultant Transfusion Physician}

2 Consultant Physician

3 National Blood Transfusion Service District General Hospital Matara

(iD) https://orcid.org/0000-0003-1890-633X
Apart from traditional indications for therapeutic apheresis there are new emerging indications in clinical practice. Here we describe few instances where it was used successfully.

\section{Propanil poisoning}

79 yr-old- patient was admitted to District General Hospital, Matara following a self ingestion of Propanil. Patient was confused and tachypnoic with mild peripheral and central cyanosis. Peripheral oxygen saturation was $76 \%$. Arterial partial pressure of oxygen was $176 \mathrm{mmHg}$. The presentation was compatible with ensuing methhemoglobinemia in propanil poisoning. Patient was treated initially with methylene blue.

As patient did not show any improvement with three cycles of methylene blue, it was decided to employ exchange transfusion as a method of removing toxic substance. Exchange transfusion had been effectively used in propanil poisoning in the past ${ }^{1,2}$. Six units of blood was removed manually and replaced with calculated amounts of fresh red cell concentrate, fresh frozen plasma and isotonic saline cyclically. Patient improved over the course of next three days.

\section{Fulminant Wilson's disease}

68-yr-old male patient presented to General Medical unit at District General Hospital Matara with evidence of hepatic encephalopathy and deep jaundice. He was a treatment defaulter with Wilson's disease. Treatment for liver cell failure with bowel decontamination, laxatives and intravenous antibiotics did not help. Correction of coagulopathy, rifaxamine and $L$ Ornithine $L$ Arginine were added to the treatment with no improvement. He underwent TPE. Around $2100 \mathrm{ml}$ of plasma was removed and replaced with calculated amounts of fresh frozen plasma, red blood cell concentrates and cryoprecipitate. Despite improvement in his biochemistry and coagulopathy patient expired after six days of intensive treatment.

In fulminant Wilson's disease acute hepatocellular necrosis release copper ions into the circulation, causing haemolysis. The use of therapeutic plasma exchange to remove copper and reduce toxic serum copper levels was studied in two patients with fulminant Wilson's disease $^{3}$. Plasma exchange allows a rapid reduction in elevated serum copper levels in these patients. . The American Society for Apheresis (ASFA) has strongly 
Clinical Practice

recommended (Category I) TPE as a treatment option for Wilson's disease ${ }^{4}$.

\section{Sickle-cell intrahepatic cholestasis}

Sickle-cell intrahepatic cholestasis (SCIC) is an uncommon complication of sickle-cell disease (SCD), which can be life-threatening if left untreated. The only effective treatment in most cases is the early onset of exchange transfusion aiming at lowering the hemoglobin $\mathrm{S}(\mathrm{HbS})$ fraction, in an attempt to minimize the intrahepatic sickling. However ASFA guidelines only recommend TPE as a category III treatment option for $\mathrm{SClC}^{4}$. A case of haemoglobin S/Beta thalassaemia with intrahepatic cholestasis was successfully treated by partial exchange blood-plasma transfusion at district general hospital Chilaw in $1995^{5}$. One unit of whole blood was removed and replaced with fresh frozen plasma (FFP) and one unit of cross-matched blood. This was done five times over the next 24 hours. The patient had shown a dramatic improvement

\section{Leptospirosis}

Leptospirosis in severe form can lead to multiorgan failure. Supportive therapy of renal, hepatic, hematologic, and central nervous system complications are important. There are cases in the literature where plasma exchange in combination with corticosteroids and intravenous immunoglobulin was beneficial in severe leptospirosis ${ }^{6,7}$. Since of late this combination of treatment has been used in leptospirosis with pulmonary complications with good effect.

\section{Thrombotic microangiopathy following snake envenomation}

The combination of acute renal failure, thrombocytopenia and microangiopathic haemolytic anaemia characterises thrombotic microangiopathy. In snake envenomation it usually occurs in association with coagulopathy ${ }^{8}$. In combination with renal failure this clinical picture is similar to haemolytic uremic syndrome (HUS) and thrombotic thrombocytopenic purpura (TTP) which usually results from a toxin causing renal endothelial damage. It is possible that a toxin in venom is causing a similar endothelial damage initiating the thrombotic microangiopathy. As in TTP thrombotic microangiopathy following snake envenomation has been treated with plasma exchange successfully ${ }^{8,9}$.

\section{Conclusion}

It is time we consider therapeutic apheresis as a treatment intervention more often. New indications are appearing in literature with compelling evidence. Practicing physicians should be informed of these indications.

\section{References}

1. Ranasinghe $P$, Dilrukshi SA, Atukorala I, Katulanda $P$, Gnanathasan A BMC Res Notes. Exchange transfusion can be life-saving in severe propanil poisoning: a case report. 2014 Oct 8;7:700. doi: 10.1186/1756-0500-7-700.

2. Eddleston $M$, Rajapakshe $M$, Roberts $D$, et al. Severe Propanil [N-(3,4-dichlorophenyl) propanamide] Pesticide Self-Poisoning. J Toxicol Clin Toxicol. 2002; 40(7): 847-854.

3. Kiss JE, Berman D, Van Thiel D. Effective removal of copper by plasma exchange in fulminant Wilson's disease. Transfusion. 1998 Apr;38(4):327-31.

4. Schwartz J, Padmanabhan A, Aqui N, et al. Guidelines on the Use of Therapeutic Apheresis in Clinical Practice Evidence-Based Approach from the Writing Committee of the American Society for Apheresis. Journal of Clinical Apheresis 31:149-338 (2016)

5. Karunatialke $\mathrm{H}$, Vithiya $\mathrm{K}$, Rajaratnam $\mathrm{M}$, Huseva $\mathrm{N}$, Ratnayake $\mathrm{H}$. Exchange transfusion for intrahepatic cholestasis due to sickle beta thalassaemia Ceylon Medical Journal 2009;.54(3):95-96

6. Meaudre E, Asencio Y, Montcriol A, Martinaud C, Graffin B, Palmier B, Goutorbe P Immunomodulation in severe leptospirosis with multiple organ failure: plasma exchange, intravenous immunoglobulin or corticosteroids? Ann Fr Anesth Reanim. 2008; 27(2):172-6

7. Sitprija V, Chusilp S Renal failure and hyperbilirubinaemia in leptospirosis. Treatment with exchange transfusion Med J Aust. 1973 Jan 27;1(4):171-3.

8. Karunatilake $\mathrm{H}$. et al.,Thrombotic microangiopathy and fibrinolysis after hump-nosed viper envenomation. Ceylon Medical Journal.2012; 57(1):45-46.

9. Isbister GK, Little M, Cull G, et al. Thrombotic microangiopathy from Australian brown snake (Pseudonaja) envenoming. Internal Medicine Journal 2007; 37: 523-8. 